\title{
Análisis de la calidad del servicio de las operadoras de telefonía móvil en la ciudad de Ambato
}

Analysis of the quality of service of mobile phone operators in the city of Ambato

Santiago Hernán Tisalema. ${ }^{1}$, Paulo César Torres. ${ }^{2}$, Nelly Janeth Cuchiparte. ${ }^{3} \&$ Byron Rodrigo Moreno. ${ }^{4}$

Recibido: 26-05-2019 / Revisado: 14-06-2019 /Aceptado: 15-07-2019/ Publicado: 06-09-2019

\begin{abstract}
.
DOI: https://doi.org/10.33262/cienciadigital.v3i3.3.770

The fourth generation mobile technology called $4 \mathrm{G}$ has been the telecommunications standard in 2019 for most mobile phone operators in Ecuador, because it has allowed to process more information and data services than $3.5 \mathrm{G}$, definitely providing a considerable increase of connection speed, consequently one of the characteristics of $4 \mathrm{G}$ relevance is undoubtedly the possibility of transmitting high resolution videos live through applications installed on smartphones and even marking the future path to the internet of things. The main objective of this work was to determine the factors that influenced the quality of the service provided by each of the mobile phone operators, for this purpose a sample of mobile phone service customers was selected, which for this purpose were students of a prestigious institution of higher education of technological level of Ambato, for this purpose surveys based on the SERVQUAL model were applied, with which the quality of the service was analyzed based on technical aspects such as: coverage, data service, voice service, technical support, and maximum and minimum speed, and with this information it was established, for example, that most clients usually activate data packets, but approximately $32 \%$ of them are satisfied with the contracted service, and this it should cause concern in the
\end{abstract}

\footnotetext{
${ }^{1}$ Instituto Superior Tecnológico Luis A. Martínez, Ambato, Ecuador, santiagohernantisalema@gmail.com

${ }^{2}$ Ambato, Ecuador, paultca@gmail.com

3 Unidad Educativa Manuel María Sánchez, nelljancut077@outlook.com

${ }^{4}$ Universidad de Especialidades Espíritu Santo, byron.moreno.dlu@gmail.com
} 
telecommunications regulation and control authorities, since it is not guaranteed that the benefits offered by the operators are concordant and efficient.

Keywords: Mobile communication, telephony, technology, services, quality.

\section{Resumen}

La tecnología móvil de cuarta generación denominada $4 \mathrm{G}$ ha sido el estándar de telecomunicaciones en el 2019 para la mayoría de las operadoras de telefonía móvil del Ecuador, debido a que ha permitido procesar mayor información y servicios de datos que $3.5 \mathrm{G}$, proporcionado definitivamente un aumento considerable de velocidad de conexión, en consecuencia una de las características de relevancia $4 \mathrm{G}$ es sin duda , la posibilidad de transmitir videos de alta resolución en vivo mediante aplicaciones instaladas en teléfonos inteligentes e incluso marcando el camino futuro hacia el internet de las cosas. El objetivo principal de este trabajo fue determinar los factores que influyeron en la calidad del servicio que suministran cada una de las operadoras de telefonía móvil, para ello se seleccionó una muestra de clientes de servicios de telefonía móvil, que para el efecto fueron estudiantes de una prestigiosa institución de educación superior de nivel tecnológico de Ambato, para ello se aplicó encuestas basadas en el modelo SERVQUAL, con lo que se analizó la calidad del servicio en función de aspectos técnicos como: la cobertura, el servicio de datos, el servicio de voz, el soporte técnico, y la velocidad máxima y mínima, y con esta información se estableció por ejemplo, que la mayoría de los clientes usualmente activa paquetes de datos, pero aproximadamente el $32 \%$ de ellos se encuentran satisfechos con el servicio contratado, y esto debería causar preocupación en las autoridades de regulación y control de las telecomunicaciones, puesto que no se está garantizando que las prestaciones que ofertan las operadoras sean concordantes y eficientes.

Palabras claves: Comunicación móvil, Telefonía, Tecnología, Servicios, Calidad.

\section{Introducción.}

En 1973 Martin Cooper introdujo la telefonía celular en los Estados Unidos con el invento del radioteléfono, luego en 1983 en los EE.UU. se regularon las reglas para la comercialización de la telefonía móvil, y tiempo después se estandarizó por varios países del mundo (Martínez, 2001).

Con la aceptación y auge da telefonía celular se desarrolla la industria de las telecomunicaciones, pues desde su introducción a principios de la década de los ochenta la telefonía celular fue diseñada únicamente para la transmisión de la voz, esto era explicable debido a las limitaciones de los equipos de telecomunicaciones de la época, pero con la evolución vertiginosa de la tecnología, se registra un desarrollo significativo en aplicaciones 
y servicios ofertados por las empresas de telefonía móvil (Rodríguez, Hernández, Torno, \& García, 2005).

Tabla 1. Evolución de la Tecnología Móvil

\begin{tabular}{|c|c|c|c|c|}
\hline $\begin{array}{c}\text { Generación } \\
\text { Móvil }\end{array}$ & Características & Tecnologías & Servicios & Período \\
\hline$\overline{1 G}$ & $\begin{array}{l}\text { Sistema analógico } \\
\text { Diseñados para transmitir solo la voz. } \\
\text { Baja capacidad de transmisión. } \\
\text { Calidad de comunicación muy baja. }\end{array}$ & $\begin{array}{l}\text { AMPS } \\
\text { TACS } \\
\text { NMT }\end{array}$ & voz & 1979 a 1989 \\
\hline $2 \mathrm{G}$ & $\begin{array}{l}\text { Sistema digital. } \\
\text { Tecnología GSM, CDMA. } \\
\text { Transmiten voz y datos. } \\
\text { Velocidades altas en voz, pero bajas en } \\
\text { datos. } \\
\text { Usan sistemas de codificación avanzados. }\end{array}$ & $\begin{array}{l}\text { D-AMPS } \\
\text { EDGE } \\
\text { GSM/GPRS } \\
\text { CDMA }\end{array}$ & $\begin{array}{l}\text { voz } \\
\text { datos }\end{array}$ & 1990 a 1999 \\
\hline$\overline{2,5 \mathrm{G}}$ & $\begin{array}{l}\text { Características extendidas de la 2G. } \\
\text { Acceso inalámbrico a internet. }\end{array}$ & $\begin{array}{l}\text { GPRS } \\
\text { HSCSD } \\
\text { EDGE } \\
\text { IS-95B }\end{array}$ & $\begin{array}{l}\text { voz } \\
\text { datos } \\
\text { video- } \\
\text { conferencia }\end{array}$ & 1999 a 2000 \\
\hline $\begin{array}{l}3 \mathrm{G} / \\
3.5 \mathrm{G}\end{array}$ & $\begin{array}{l}\text { Comunicación por acceso al Internet. } \\
\text { Aparición de aplicaciones multimedia. } \\
\text { Velocidad de transmisión de hasta } 384 \\
\text { Kbps. } \\
\text { Mejores seguridades. } \\
\text { Limitada movilidad de los usuarios. }\end{array}$ & $\begin{array}{l}\text { UMTS } \\
\text { IMT-2000 } \\
\text { CDMA2000 } \\
\text { EV - DO } \\
\text { WCDMA / } \\
\text { HSPA }\end{array}$ & $\begin{array}{l}\text { voz } \\
\text { datos } \\
\text { multimedia }\end{array}$ & 2001 a 2009 \\
\hline$\overline{4 \mathrm{G}}$ & $\begin{array}{l}\text { Velocidad Tx: } 300 \mathrm{Mb} / \mathrm{s} \\
\text { Internet banda ancha }\end{array}$ & LTE & Servicios IP & 2010 a 2019 \\
\hline
\end{tabular}

Fuente: (Barreno, Carrión, \& Tenedora, 2016) 
ISSN: 2602-8085

www.cienciadigital.org

Vol. 3, N³.3, p. 59 - 76, septiembre, 2019

\section{Telefonía móvil en el Ecuador}

El organismo rector encargado de controlar el uso del espectro electromagnético y por consiguiente las telecomunicaciones y redes de datos en el Ecuador desde el año 2015 es la Agencia de Regulación y Control de las Telecomunicaciones conocida como "ARCOTEL", la cual cuenta con ocho sub - agencias en todo el país. Así se puede acceder a cualquiera de las coordinaciones zonales en Quito, Riobamba, Portoviejo, Guayaquil, Cuenca, Galápagos, Loja, Lago Agrio, siendo su oficina matriz en Quito. Su función principal es la de monitorear todas las redes de telecomunicaciones para garantizar un adecuado uso del espectro en cualquiera de las aplicaciones tecnológicas de comunicaciones, en especial las comunicaciones móviles.

En la página de ARCOTEL periódicamente se publican informes técnicos a los cuales el usuario puede acceder sin ningún inconveniente, para el presente caso de estudio dicha agencia tiene registrado y reconoce para el año 2019 a tres operadoras que proporcionan servicios de telefonía móvil, las cuales son:

- TELECSA S.A “Telecomunicación Móvil del Ecuador S.A.” que funciona a partir de abril del 2003, en la actual es conocida como la Corporación Nacional de Telecomunicaciones CNT S.A.

- CONECEL S.A., conocida con la marca CLARO, autorizada desde agosto 2008.

- OTECEL S.A., popularmente conocida como MOVISTAR, autorizada desde noviembre 2008 (Albornoz \& Agüero, 2011). También posee otra marca conocida como Tuenti.

\section{Calidad del Servicio}

Es el resultado de comparar las expectativas del consumidor entre el servicio que recibe, es decir la fidelización del cliente depende de la calidad del servicio, siempre y cuando este se encuentre satisfecho (Matsumoto Nishizawa, 2014). 
La calidad del servicio es un tema que se debe manejar con responsabilidad desde el enfoque administrativo para cualquier entidad, puesto que un usuario plenamente satisfecho se traduce en un cliente leal que está dispuesto a emitir comentarios positivos a otros clientes (Moliner, 2001).

Para (Restrepo, Restrepo, \& Estrada, 2006) actualmente el servicio está enmarcado en estrategias para atraer y sobre todo mantener la fidelidad del consumidor, lo que se traduce buenos resultados de la gestión administrativa de las relaciones con el cliente con el propósito integral de satisfacer sus necesidades.

\section{SERVQUAL}

El modelo SERVQUAL es la herramienta más utilizada para medir la calidad del servicio, esta se puede aplicar a diferentes áreas de la empresa de diferentes tipos, permitiendo así evaluar por separado las expectativas y las percepciones del cliente. En sus inicios este modelo se basó en 10 aspectos, dependiendo del tipo de servicio prestado, los aspectos analizados eran: Elementos tangibles, Fiabilidad, Capacidad de respuesta, Profesionalidad, Cortesía, Credibilidad, Seguridad, Accesibilidad, Comunicación, Compresión del cliente (Matsumoto Nishizawa, 2014).

Para (Calixto, y otros, 2011) SERVQUAL es una escala multidimensional, constituida a su vez por dos factores considerados como: expectativas y percepciones del cliente.

Figura 1. Calidad de Servicio
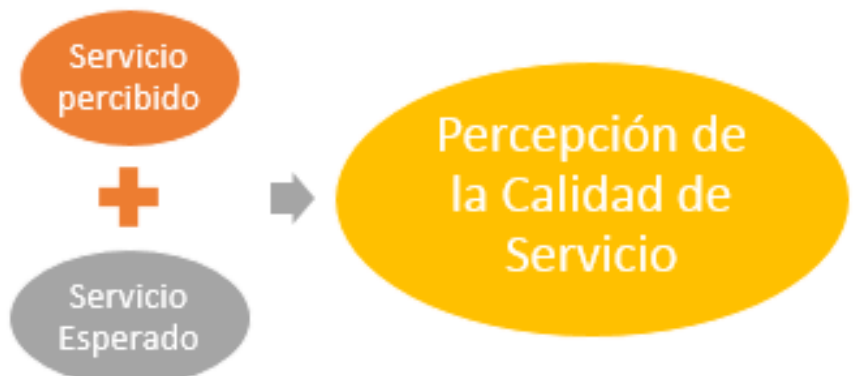

Fuente: (Duque, 2005) 
Después de diversos análisis y revisiones del modelo SERVQUAL, se puede reducir los diez aspectos que implica el modelo a cinco, los cuales son:

Confianza o empatía, es entender la preocupación del usuario de forma individual. Según (Moya, Herrero, \& Bernal, 2010). La respuesta empática incluye la capacidad para comprender al otro y ponerse en su lugar a partir de lo que se observa.

1. Fiabilidad, es la capacidad en la que se entrega el servicio ofertado es decir la precisión y solides del mismo.

2. Capacidad de respuesta, es prestar ayuda cuando el usuario lo solicite de forma rápida.

3. Se le conoce también como capacidad de absorción, es un conjunto de estrategias para que las empresas obtengan, comprendan y exploten de cierta forma un mejor conocimiento del exterior y respondan asertivamente ante ello (Demuner, Urbano, \& Ibarra, 2018).

4. Responsabilidad, es el grado de conocimientos que tiene el empleado de los productos y/o servicios ofertados, esto permite crear un ambiente de confianza y credibilidad.

5. Tangibles, es la apariencia de la institución, del equipamiento, personal, etc. (Morales, Hernández Mendo, \& Blanco, 2009).

Figura 2. Modelo SERVQUAL

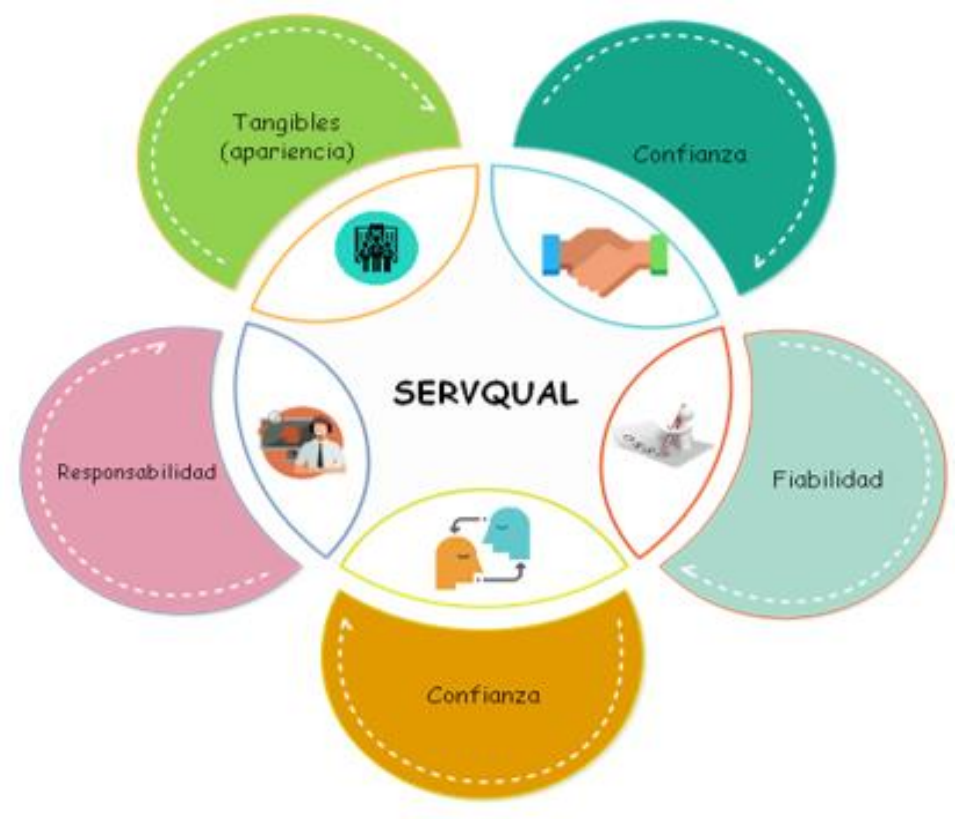

Fuente: (Duque, 2005) 


\section{Redes Sociales}

Las redes sociales permiten a las personas la creación de nuevos entornos de comunicación y socialización, sin importar la ubicación geográfica donde se encuentren conectados, el sexo, la edad, el estado civil, etc. basta con compartir un interés en común, también es permitido a las empresas que puedan crecer en el mercado y ganancias, a través de la creación de campañas publicitarias que pueden llegar a más personas, en menos tiempo y menor costo (Bernal Bravo \& Angulo Rasco, 2013).

En la última década las redes sociales constituyen un medio de comunicación mediante el cual las personas manifiestan su libertad de expresión e incluso se han utilizado masivamente en los denominados grupos, y de ello las instituciones comerciales e industriales han sacado provecho en relación directa a difundir su imagen institucional o corporativa de una manera rápida, efectiva, con menor incidencia en recursos humanos y lo más interesante es que se reduce significativamente los costos publicitarios, permitiendo mejorar la rentabilidad de la entidad, por lo dicho, las tres operadoras del país antes mencionadas, han tenido que implementar nuevos planes de datos que incluyen redes sociales ilimitadas para intentar en algo mejorar la sensación de insatisfacción de sus clientes, sin embrago el problema radica fundamentalmente en el número de equipos de interconexión de las redes denominados radio bases, es así que, CONECEL posee la mejor cobertura debido a que ha implementado el mayor número de radio bases alrededor del país.

\section{Metodología}

Esta investigación analiza los factores que influyen en la Calidad del Servicio de las empresas que proveen telefonía móvil en el Ecuador en la ciudad de Ambato, para ello se realizó el análisis en los estudiantes del Instituto Superior Tecnológico "Luis A. Martínez”, donde participaron alrededor de 181 estudiantes entre hombres y mujeres, los cuales se encontraban matriculados y asistían regularmente.

El Instituto Superior Tecnológico "Luis A. Martínez" tiene cuatro especialidades las cuales son:

- Administración de Empresas mención Contabilidad y Auditoría 
- Administración Financiera

- Análisis de Sistemas

- Redes y Telecomunicaciones.

La presente investigación se desarrolló en tres fases:

1) Estudio bibliográfico.

2) Recolección de datos.

3) Análisis de los factores y su relación con la calidad de servicio de la Telefonía Móvil 4G.

En la revisión bibliográfica se consultaron libros físicos y digitales, base de datos de diferentes repositorios digitales de universidades y páginas web relacionadas al tema investigado.

Para la recolección de datos se elaboró un cuestionario con preguntas de selección múltiple de una sola respuesta y se utilizó varias escalas de Likert, este cuestionario fue validado a través del programa IBM SPSS Statistics en su versión 23, el método que se aplicó para la validación fue el Alfa de Cronbach en mismo que permite determinar el grado de consistencia de un instrumento de evaluación y qué nivel de confianza tendrá al ser usado (Bojórquez Molina, López Aranda, Hernández Flores, \& Jiménez López, 2013).

Para validar el cuestionario se realizó una prueba piloto, el mismo que obtuvo como coeficiente de Alfa de Cronbach de 0,995 lo que significa que es excelente, lo cual nos indica que no se va a tener problemas de consistencia con los resultados al aplicar el cuestionario a los estudiantes del instituto.

Tabla 2. Grado de fiabilidad a través del coeficiente de alfa de Cronbach

\begin{tabular}{cc}
\hline Coeficiente Alfa Cronbach & Grado de Fiabilidad \\
\hline$>0,9$ & Excelente \\
$>\mathbf{0 , 8}$ & Bueno \\
$>\mathbf{0 , 7}$ & Aceptable \\
$>\mathbf{0 , 6}$ & Cuestionable \\
$>\mathbf{0 , 5}$ & Pobre \\
$<\mathbf{0 , 5}$ & Inaceptable \\
\hline
\end{tabular}

Fuente: (George \& Mallery, 2003) 
Tabla 3. Fiabilidad del Cuestionario Aplicado

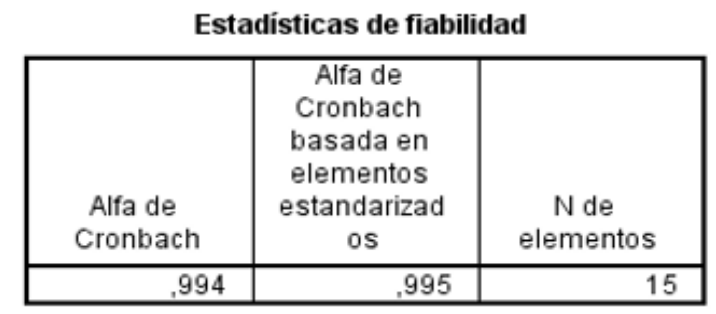

Fuente: IBM SPSS Statistics 23

El cuestionario se aplicó a la muestra calculada de la población existente de 339 estudiantes matriculados en el Instituto Superior Tecnológico "Luis A. Martínez", los cuales estaba compuesto por 179 mujeres y 160 hombres.

Figura 3. Población de Estudiantes matriculados en el Instituto Superior Tecnológico "Luis A. Martínez

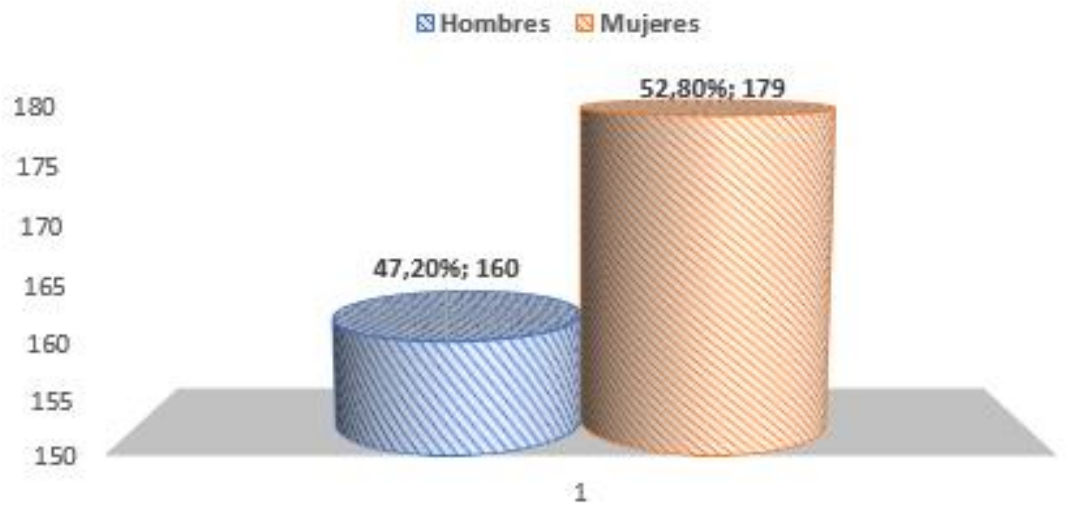

Fuente: Trabajo de campo

Para calcular la muestra se aplicó la fórmula para una población finita, y como normalmente el nivel de confianza es del 95\% para que la información recopilada sea fiable, en consecuencia, el margen de error estándar aplicado fue del 5\%, son ello se obtuvo como resultado 180,33433 lo que equivale eventualmente a 180 estudiantes, a los cuales se aplicó las encuestas (Gallego, 2004).

Donde se obtiene el siguiente resultado:

$$
n=\frac{Z^{2} * p * q * N}{\left(e^{2} *(N-1)\right)+Z^{2} * p * q}
$$


De la formula anterior se puede deducir lo siguiente:

- $\quad$ Nivel de confianza (95\%): $Z=1,96$

- Error Muestral: e $=5 \%$

- Población: $\mathrm{N}=339$

- Probabilidad de éxito: $\mathrm{p}=0,5$

- $\quad$ Probabilidad de fracaso $(1-p)=q=0,5$

$$
n=\frac{1,96^{2} * 0,5 * 0,5 * 339}{\left(0,05^{2} *(339-1)\right)+1,96^{2} * 0,5 * 0,5}=180,33433
$$

El último paso realizado fue analizar e interpretar los resultados de las encuestas aplicadas, para determinar cuál fue la relación que existe entre la calidad de servicio y las operadoras de telefonía móvil.

\section{Resultados}

De acuerdo al estudio efectuado con la muestra, es decir los estudiantes de carreras de tecnología superior en Redes y Telecomunicaciones, Análisis de Sistemas , Administración de Empresas mención Contabilidad y Auditoría , y Administración Financiera del Instituto Superior Tecnológico "Luis A. Martínez” con una población aproximada de 339 estudiantes entre hombres y mujeres, y dado que aproximadamente el $90 \%$ de ellos posee teléfonos inteligentes, sin tomar en cuenta la operadora se puede mencionar que aproximadamente el $53 \%$ de los educandos clientes de telefonía móvil, activan usualmente paquetes de datos con frecuencia semanal, por otro lado se puede destacar también que alrededor del $35 \%$ de ellos indican que el servicio que han contratado a veces satisface sus requerimientos y expectativas; el 30\% manifiesta que cuando tienen algún problema técnico referente a por lo general a conexión a internet la atención al cliente es medianamente satisfactoria. Teniendo en cuenta dichos resultados se puede inferir como conclusión de que apenas el $32 \%$ de los clientes están conformes con su operadora. 
Figura 4. Frecuencia de activación de plan de datos móviles

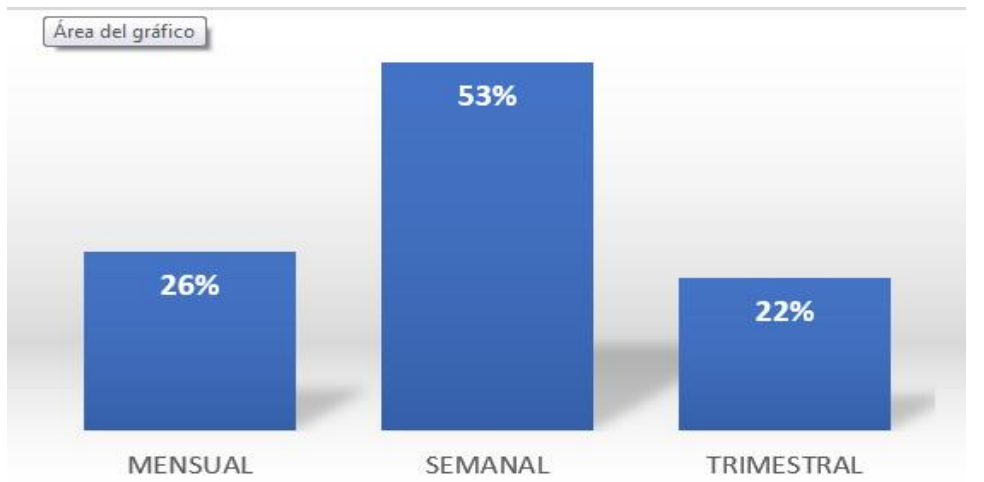

Fuente: Trabajo de campo

Se puede ratificar la preferencia de los estudiantes en un $43.89 \%$ por el uso frecuente de las redes sociales como medio de comunicación, esto se pude explicar debido a que dichas aplicaciones de interacción social permiten dejar a un lado las condiciones sociales y económicas ,e incluso su entorno en general es más eficiente que las aplicaciones o herramientas insertadas en celulares o equipos de usuario final de telecomunicaciones consideradas tradicionales desde el inicio de las comunicaciones móviles, es decir las llamadas y mensajes de texto. Con las ventajas de $4 \mathrm{G}$ los mensajes de texto prácticamente han quedado relegados.

Todo lo antes mencionado se contrasta con la necesidad insatisfecha del cliente desde el punto de vista de que los dispositivos móviles tengan una conexión estable y permanente hacia el internet.

Figura 5. Uso del servicio de telefonía móvil

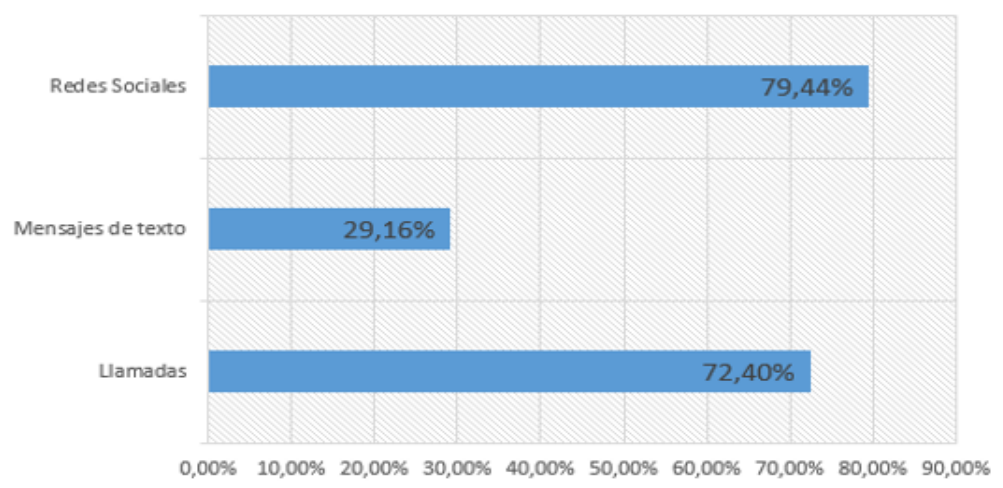

Fuente: Trabajo de campo 
Los clientes casi siempre pueden utilizar los servicios contratados, pero en la mayoría de los casos no se encuentra disponible, esto es debido a la cobertura o a las fallas que presentan los sistemas de la operadora de telefonía móvil.

Figura 6. Confianza y satisfacción de la operadora de telefonía

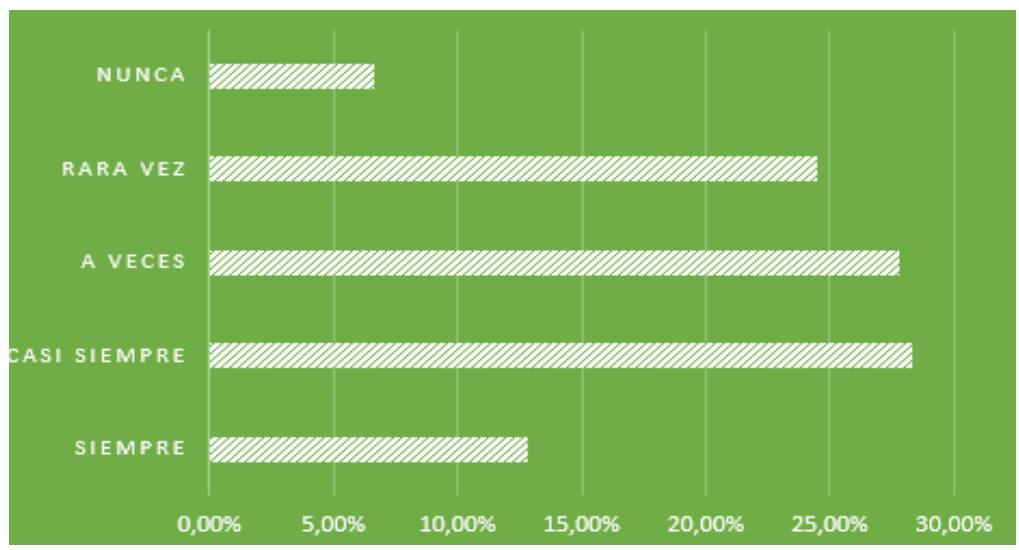

Fuente: Trabajo de campo

Las empresas operadoras de telefonía móvil en la mayoría de los casos no es clara en la información entregada al momento de contratar el servicio, por lo que los clientes se ven afectados con costos adicionales, también el soporte técnico no es el apropiado, porque deben esperar mucho tiempo para poder ser atendido y en ocasiones no es resuelto.

Figura 7. Costos de Servicios y Soporte Técnico
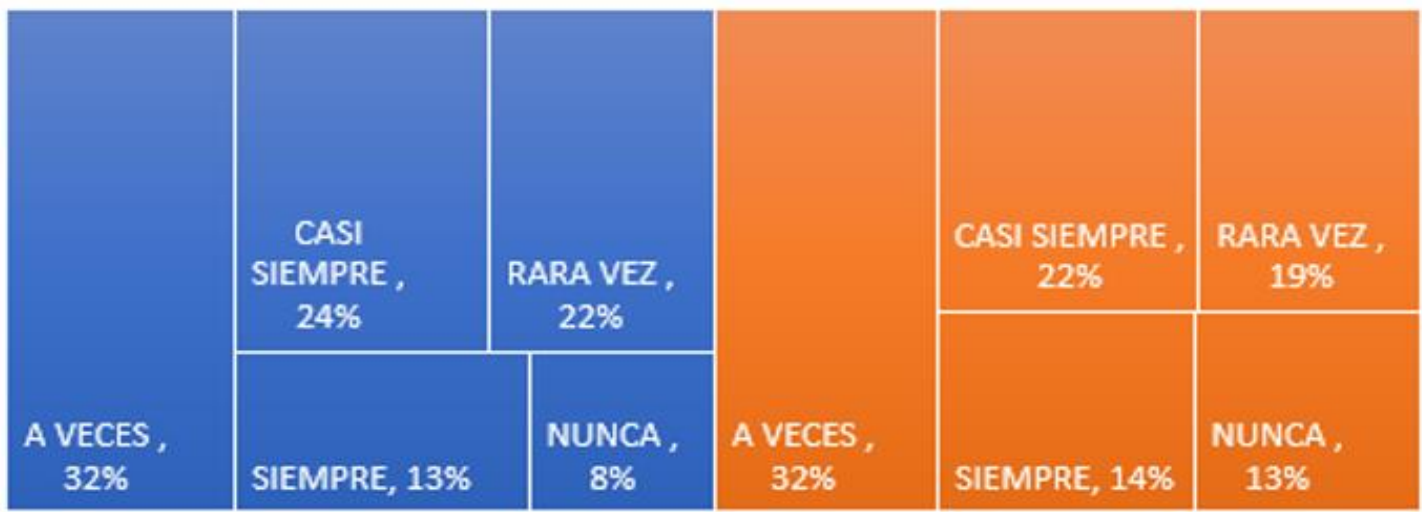

Las solicitudes de soporte técnico son resueltas en el menor tiempo.

Es clara la información sobre las modificaciones en los cobros del servicio.

Fuente: Trabajo de campo 


\section{Cobertura 4G en Ecuador}

Según datos (Ministerio de Telecomunicaciones y de la sociedad de la información, 2019) las redes móviles en Ecuador gozan una aceptable cobertura principalmente en los centros densamente poblados como: Quito, Guayaquil, Manta, Cuenca, donde se registra que el acceso a la tecnología $4 \mathrm{G}$, incrementa frecuentemente a tal punto que existe una cobertura geográfica equivalente de alrededor del $45 \%$, lo que evidencia que la realidad del sector de las telecomunicaciones en el país.

Desde que se insertó la cuarta generación de las telecomunicaciones móviles se ha masificado el uso del internet mediante equipos móviles, como muestra de este hecho hasta septiembre del 2019 se registraron más de nueve millones de cuentas de Internet móvil, esto significó un incremento numérico de 386000 más de las reportadas hasta marzo de 2018 (Agencia de Regulación y Control de las Telecomunicaciones, 2019).

\section{Velocidad de conexión}

La velocidad de conexión representa básicamente el promedio de información que se transmite entre dos dispositivos electrónicos de comunicación en función del tiempo y a lo largo de una red de telecomunicaciones (Wikipedia, 2019).

Dado que la velocidad de conexión se considera una magnitud física se mide comúnmente en bits por segundo. En este punto se ha decidido realizar una breve comparación de velocidad de conexión entre 3G que oscilaba entre 64 y 384 kbps (kilo bits por segundo), la velocidad para 3.5G era aproximadamente 22Mbps, y finalmente 4G-LTE (1700 MHz), con una velocidad de bajada 75Mbps y tasa de subida de 25 Mbps, según (elhacker.NET, 2019)

La velocidad de conexión es un factor muy importante en la aceptación del servicio móvil y sin embargo alrededor del 95\% de los estudiantes desconoce las características de velocidad que contratan, esto obedece a que las operadoras generalizan las propuestas de los paquetes de datos que comercializan, así por lo general un cliente adquiere el servicio, más por las aplicaciones relacionadas a redes sociales, que por selección de las características técnicas de la red celular. 


\section{Discusión}

El estudio proyectaba de cierta forma determinar la incidencia de factores técnicos como la confianza, fiabilidad, capacidad de respuesta, responsabilidad y la imagen empresarial en la calidad del servicio que oferta la operadora de telefonía móvil mediante el modelo SERVQUAL, y luego de la aplicación de encuetas de satisfacción del cliente se pudo evidenciar el grado de satisfacción del usuario con la operadora, iniciando por la concordancia entre los servicios que se acuerda y la precisión o efectividad del mismo, y sobre todo observar el comportamiento de los clientes en el caso de que existan valores adicionales que le perjudiquen al usuario.

Los organismos de control de las telecomunicaciones aducen que existe desarrollo significativo en el sector, dado que se registra un incremento de aproximadamente el 4\% usuarios en comparación al 2018, sin embargo el grado de satisfacción de los clientes según los resultados obtenidos en la muestra de estudiantes del Instituto "Luis A. Martínez" es medianamente aceptable, a pesar de que las características de la tecnología 4G deberían haber incrementado exponencialmente los usuarios móviles como es el caso de otros países de la región.

A pesar de las dificultades y la intermitencia que las redes móviles presentan en la actualidad la mayoría de los usuarios de las operadoras de telefonía móvil se mantienen fieles, lo que no significa necesariamente que el servicio que oferta las operadoras haya mejorado, más bien este fenómeno se explica por la necesidad cotidiana de comunicación antes que por la percepción de un servicio adecuado y de calidad.

\section{Conclusiones.}

- La calidad de servicio es el eje principal para el crecimiento y desarrollo de cualquier entidad o empresa, mediante la evaluación de la calidad del servicio por el modelo SERVQUAL, se puede evidenciar falencias entre las prestaciones ofertadas y las que realmente ocurren. En este caso de estudio se pudo localizar deficiencias en la atención al cliente e inconsistencias en cuanto al servicio ofertado por las empresas operadoras de servicios de telecomunicaciones móviles, además el servicio técnico se considera 
inadecuado, todo ello se podría optimizar mediante una medición de satisfacción en base a la mejora de la cobertura y velocidad.

- Se puede decir que las operadoras de telefonía móvil en Ecuador a pesar de haber migrado a 4G, deben invertir en aumentar su cobertura (radio bases) para reducir la intermitencia de la señal en función de mejorar la calidad de servicio y la agilidad del soporte técnico, así captar la confianza del cliente.

- Los servicios contratados por el estudiante son relativamente costosos si se toma en cuenta la poca calidad del servicio que se percibe y las fallas técnicas, sin embargo, la mayoría de abonados móviles no acostumbra emitir quejas o reclamos debido a la desinformación que existe sobre leyes que regulan el uso del espectro electromagnético.

- Se pude mencionar que el uso de datos y aplicaciones compatibles con el estándar 4G, requieren que el dispositivo móvil soporte el tráfico de datos con un buen ancho de banda, sin embrago la necesidad de comunicación por medio de redes sociales provoca ventajas en cuanto a la difusión y de comercialización de productos de una manera más eficiente y efectiva, en concordancia con las exigencias de los usuarios finales.

\section{Referencias bibliográficas.}

Agencia de Regulación y Control de las Telecomunicaciones. (04 de 09 de 2019). Crecimiento de Internet móvil en Ecuador. Obtenido de Crecimiento de Internet móvil en Ecuador: http://www.arcotel.gob.ec/crecimiento-de-internet-movil-enecuador/

Albornoz, M. B., \& Agüero, A. (2011). El estado de la banda ancha en Ecuador. Diálogo Regional sobre Sociedad de la Información, 1-44.

Barreno, D., Carrión, D., \& Tenecora, I. (noviembre de 2016). Evolución de la tecnología móvil. Camino a 5G. Revista Contribuciones a las Ciencias Sociales. Obtenido de http://www.eumed.net/rev/cccss/2016/04/5G.html

Bernal Bravo, C., \& Angulo Rasco, F. (2013). Interacciones de los jóvenes andaluces en las redes sociales. Revista Científica de Educomunicación, 25-30.

Bojórquez Molina, J. A., López Aranda, L., Hernández Flores, M. E., \& Jiménez López, E. (2013). Utilización del alfa de Cronbach para validar la confiabilidad de. Eleventh 
LACCEI Latín American and Caribbean Conference for Engineering and Technology, 1-9.

Calixto, M., Okino, N., Hayashida, M., Costa, I., Trevizan, M., \& de Godoy, S. (Julioseptiembre de 2011), Escala SERVQUAL: validación en población mexicana. Texto \& Contexto Enfermagem, 20(3), 326-333. Obtenido de http://www.redalyc.org/articulo.oa?id=71421157014

Demuner, M., Urbano, s., \& Ibarra, M. (2018). Capacidad de respuesta y capacidad de absorción. Estudio de empresas manufactureras en México. Nóesis. Revista de Ciencias Sociales y Humanidades, 27(53-2), 61-77. Obtenido de http://www.redalyc.org/articulo.oa?id=85955218004

Duque, E. (2005). Revisión del concepto de calidad del servicio y sus modelos de medición. INNOVAR, 15, 64-80.

elhacker.NET. (04 de 09 de 2019). Velocidad Conexiones GSM, GPRS, 3G+, 4G, LTE, UMTS, HSDPA. Obtenido de ¿Cuáles son las diferencias entre E, GPRS, 3G, 4G, 5G? Diferentes velocidades: https://www.elhacker.net/diferencias-conexiones-3ghsdpa-umts.html

Gallego, C. F. (2004). Cálculo del tamaño de la muestra. Matronas profesión, 5-13.

George, D., \& Mallery. (2003). Alfa de Cronbach y consistencia interna de los ítems de un instrumento de medida. Revista de estudios Interdisciplinarios en Ciencias Sociales, 3-9.

Martínez, E. (2001). La evolución de la telefonía móvil. Revista Red, 1-6.

Matsumoto Nishizawa, R. (2014). Desarrollo del Modelo Servqual para la medición de la calidad del servicio en la empresa de publicidad Ayuda Experto. Perspectivas, 181 209.

Ministerio de Telecomunicaciones y de la sociedad de la información. (31 de agosto de 2019). Cobertura del 4G aumenta en el Ecuador. Obtenido de https://www.telecomunicaciones.gob.ec/cobertura-del-4g-aumenta-en-ecuador/

Moliner, C. (2001). Calidad de Servicio y Satisfacción del cliente. Revista de Psicología del Trabajo y de las Organizaciones, 17(2), 233-235. Obtenido de http://www.redalyc.org/articulo.oa?id=231324550006 
Morales, V., Hernández Mendo, A., \& Blanco, Á. (2009). Evaluación de la calidad en organizaciones deportivas: adaptación del modelo SERVQUAL. Revista de Psicología del deporte, 137-150.

Moya, L., Herrero, N., \& Bernal, M. (2010). Bases neuronales de la empatía. Rev Neurol, 50(2), 89-100.

Restrepo, C., Restrepo, L., \& Estrada, S. (12 de 2006). Enfoque estratégico del servicio al cliente. Scientia Et Technica, XII (32), 289-294. Obtenido de http://www.redalyc.org/articulo.oa? $\mathrm{id}=84911652051$

Rodríguez, O., Hernández, R., Torno, L., \& García. (2005). Telefonía móvil celular: origen, evolución, perspectivas. Ciencias Holguín, 1-8.

Wikipedia. (13 de 07 de 2019). Velocidad de conexión. Obtenido de https://es.wikipedia.org/wiki/Velocidad_de_conexi\%C3\%B3n

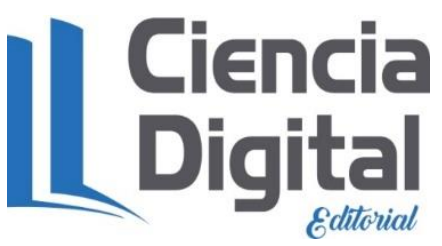




\section{PARA CITAR EL ARTÍCULO INDEXADO.}

Tisalema, S., Torres, P., Cuchiparte, N., \& Moreno., B. (2019). Análisis de la calidad del servicio de las operadoras de telefonía móvil en la ciudad de Ambato. Ciencia Digital, 3(3.3), 59-79. https://doi.org/10.33262/cienciadigital.v3i3.3.770

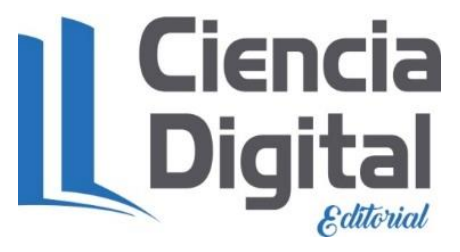

El artículo que se publica es de exclusiva responsabilidad de los autores y no necesariamente reflejan el pensamiento de la Revista Ciencia Digital.

El artículo queda en propiedad de la revista y, por tanto, su publicación parcial y/o total en otro medio tiene que ser autorizado por el director de la Revista Ciencia Digital.
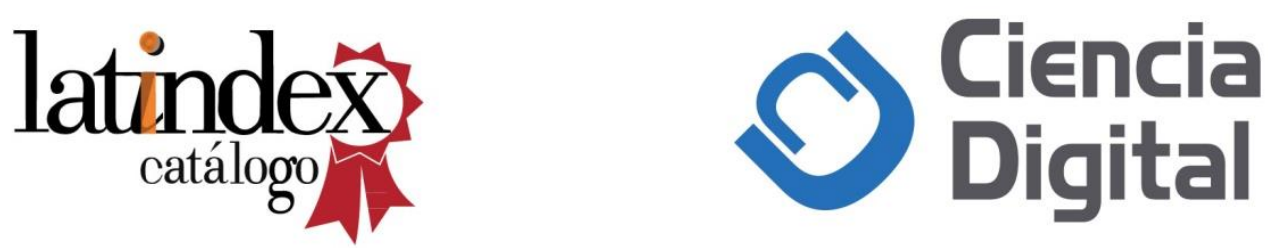